\title{
TEOR E COMPOSIÇÃo DO ÓLEO ESSENCIAL DE CINCO ACESSOS DE MENTRASTO
}

\section{Henrique Guilhon de Castro*}

Laboratório de Melhoramento Genético Vegetal, Universidade Estadual do Norte Fluminense,

Av. Alberto Lamego, 2000, 28015-620 Campos dos Goytacazes - RJ

\section{Luiz Orlando de Oliveira}

Departamento de Bioquímica e Biologia Molecular, Universidade Federal de Viçosa, 36571-000 Viçosa - MG

Luiz Cláudio de Almeida Barbosa

Departamento de Química, Universidade Federal de Viçosa, 36571-000 Viçosa - MG

Francisco Affonso Ferreira e Derly José Henriques da Silva

Departamento de Fitotecnia, Universidade Federal de Viçosa, 36571-000 Viçosa - MG

Paulo Roberto Mosquim

Departamento de Biologia Vegetal, Universidade Federal de Viçosa, 36571-000 Viçosa - MG

Evandro Afonso Nascimento

Instituto de Química, Universidade Federal de Uberlândia, 38400-902 Uberlândia- MG

Recebido em 11/2/03; aceito em 13/8/03

\begin{abstract}
CONTENT AND COMPOSITION OF THE ESSENTIAL OIL OF FIVE ACCESSES OF MENTRASTO. This study aimed to analyse the content and the composition of the essential oil of five accesses of mentrasto (Ageratum conyzoides). Five accesses of mentrasto with three repetitions were used. The accesses were obtained in Mariana-MG (AMA), Piranga-MG (API), Visconde do Rio Branco (ARB) and Viçosa-MG (AVB and AVP). The essential oil was obtained by hydrodistillation and the identification of the oil components by CG and GC/MS. The ARB access presented the higher essential oil content, that is $0.70 \%(\mathrm{P}<0.05)$. Eleven chemical compounds were identified. The precocene I was the main constituent in the access API, and the precocene II was the main constituent in the accesses AMA, ARB, AVB and AVP.
\end{abstract}

Keywords: Ageratum conyzoides; essential oil; genetic resources of plants.

\section{INTRODUÇÃO}

A variação genética em populações naturais de plantas e animais é a base da sua resistência perante as pressões do ambiente, sendo a matéria-prima da seleção natural. As plantas que ocorrem ao longo de um gradiente ambiental, por exemplo, variam também quanto à constituição genética e atividade fisiológica, condicionadas pelo processo de seleção natural; embora pertencendo à mesma espécie, podem responder de modo muito diferente a dado grau de tensão ambiental. A diversidade genética envolve, portanto, o metabolismo dos organismos e seus produtos ${ }^{1,2}$.

Estudos em quimiotaxonomia consistem na investigação de compostos químicos que ocorrem em plantas, com o objetivo de evidenciar ou não a relação entre elas, devendo a ênfase ser dada aos compostos predominantes da espécie ${ }^{3,4}$. A padronização das épocas de colheita, da parte colhida e do cultivo sob as mesmas condições ambientais auxilia na identificação de variedades que apresentam diferenças na sua composição química ${ }^{5}$.

Os óleos essenciais são misturas complexas que podem conter 100 ou mais compostos orgânicos. Seus constituintes podem pertencer às mais diversas classes de compostos, porém os terpenos e os fenilpropenos são as classes de compostos mais comumente encontradas. Os terpenos encontrados com maior frequiência nos óleos essenciais são os monoterpenos e sesquiterpenos, bem como os diterpenos, constituintes minoritários dos óleos essenciais ${ }^{6-10}$.

*e-mail: hguilhon@uenf.br
Os compostos predominantes do óleo essencial de Ageratum conyzoides são os cromenos, principalmente precoceno I e precoceno $\mathrm{II}^{11}$, que causam metamorfose prematura em diversas espécies de insetos, levando à formação de adultos estéreis ${ }^{12}$.

Estudos realizados com o óleo essencial de A. conyzoides em ratos mostraram que ele possui significativa atividade analgésica, antiinflamatória e antipirética, não sendo observada toxicidade gástrica $^{13,14}$.

O mentrasto (Ageratum conyzoides L.) é planta nativa da América com adaptação a diversas condições ambientais, estabelecendo-se em várias regiões de clima tropical e subtropical do mundo. Planta considerada invasora em cerca de 50 países, tem valor ornamental e, na Malásia, é usada como forrageira para cabras, bovinos e muares $^{15,16}$.

Ageratum conyzoides apresenta uso medicinal difundido pela população no Brasil e em outros países. A droga vegetal denominada mentrasto (Ageratum conyzoides) tem tido seu consumo aumentado, a partir de sua inclusão na lista da Central de Medicamentos e subseqüente verificação de sua eficácia como analgésico e antiinflamatório ${ }^{17}$. Embora comprovada sua atividade, o vegetal não se acha ainda em fase de cultivo racional. A droga que abastece o mercado de São Paulo e Rio de Janeiro e, por extensão, todo o Brasil é proveniente do extrativismo ${ }^{18}$.

O objetivo deste trabalho foi analisar o teor e a composição do óleo essencial de cinco acessos de Ageratum conyzoides, provenientes de diferentes origens geográficas, mas cultivados sob as mesmas condições ambientais. De forma mais específica, buscou-se responder às seguintes questões: 1) Os acessos estudados apresentariam variação no teor de óleo essencial? 2) Haveria variação na composi- 
ção do óleo essencial? 3) Quais os compostos predominantes do óleo essencial?

\section{RESULTADOS E DISCUSSÃO}

\section{Teor do óleo essencial}

No acesso obtido no município de Visconde do Rio Branco- ARB foi verificado o maior valor de teor do óleo essencial, 0,70\% (Tabela 1), e no acesso obtido no município de Piranga- API o menor valor, $0,48 \%$.

Tabela 1. Valores médios do teor de óleo essencial, em \% da planta desidratada, de cinco acessos de Ageratum conyzoides

\begin{tabular}{cc}
\hline Acessos & Teor de óleo essencial $(\%)$ \\
\hline AMA & $0,49 \mathrm{~b}$ \\
API & $0,48 \mathrm{~b}$ \\
ARB & $0,70 \mathrm{a}$ \\
AVB & $0,49 \mathrm{~b}$ \\
AVP & $0,51 \mathrm{~b}$
\end{tabular}

Médias seguidas pela mesma letra na coluna não diferem entre si pelo teste de Tukey $(\mathrm{P}>0,05)$. AMA $=$ acesso coletado em MarianaMG; API = acesso coletado em Piranga-MG; ARB = acesso coletado em Visconde do Rio Branco-MG; AVB e AVP = acessos coletados em Viçosa-MG.

A constituição genética das plantas influencia a produção de determinados metabólitos, e diferentes variedades de espécies de plantas medicinais podem conter diferentes teores de óleo essencial. A hortelã, por exemplo, possui variedades que são mais ricas em óleo essencial, contendo alto teor de mentol ${ }^{19,20}$.

\section{Composição do óleo essencial}

Ocorreu variação no número de compostos do óleo essencial presentes nos acessos de mentrasto. Foi obtido no acesso API o maior número de compostos, e nos acessos de Viçosa (AVB e AVP) os menores valores em número de compostos (Tabela 2 ).

Os compostos identificados no óleo essencial dos acessos de mentrasto podem ser divididos em: monoterpenos, sesquiterpenos, cromenos e fenilpropenos, mostrados na Tabela 2 e na Figura 1.

Foram identificados dois compostos majoritários, ambos cromenos: o precoceno I e o precoceno II. O precoceno I foi o com-

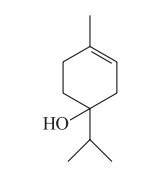

Terpinen-4-ol

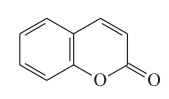

Cumarina
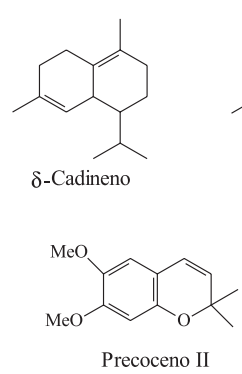

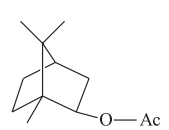

Acetato de bornila

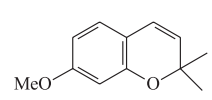

Precoceno I

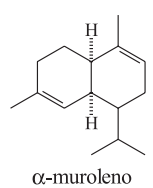

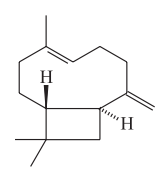

(E)-Cariofileno
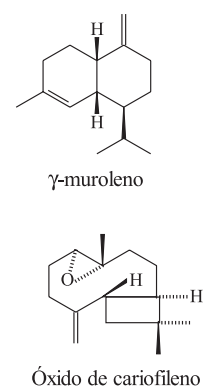

Óxido de cariofileno

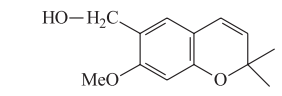

6-(hidroximetil)-7-metoxi-2,2-dimetil-2H-cromeno
Figura 1. Fórmulas estruturais dos constituintes presentes no óleo essencial da parte aérea de Ageratum conyzoides (identificados por cromatografia gasosa acoplada ao espectrômetro de massas)

posto majoritário no acesso API, e o precoceno II, o composto majoritário nos outros acessos. Outro cromeno identificado, relatado pela primeira vez nessa espécie, foi o 6-hidroximetil-7-metoxi-2,2-dimetil$2 \mathrm{H}$-cromeno, constituinte minoritário não encontrado no acesso AVB.

Resultados semelhantes foram encontrados em plantas de Ageratum conyzoides originárias de países da Ásia e África, encontrando como constituintes majoritários os cromenos (precoceno I, precoceno II e 6-vinil-7-metoxi-2,2-dimetilcromeno) ${ }^{21-23}$.

Os monoterpenos identificados foram o terpinen-4-ol e o acetato de bornila. Os sesquiterpenos identificados foram o $(E)$-cariofileno, o $\gamma$-muroleno, o $\delta$-cadineno, o $\alpha$-muroleno e o óxido de cariofileno. Entre os terpenos, o $(E)$-cariofileno foi o composto encontrado em maior concentração, fato também observado em outros trabalhos ${ }^{21,23}$.

O $\delta$-cadineno, composto presente somente no acesso API, é precursor do gossipol, composto que atua na defesa química das plan$\operatorname{tas}^{24}$.

Em relação aos fenilpropenos, identificaram-se as cumarinas. A maior concentração de cumarina foi encontrada no acesso API $(3,94 \%)$, sendo esta ausente no acesso AVB.

Tabela 2. Concentração relativa (\%), obtida por cromatografia gasosa, dos constituintes do óleo essencial da parte aérea de cinco acessos de mentrasto (Ageratum conyzoides)

\begin{tabular}{|c|c|c|c|c|c|c|c|c|c|c|c|c|}
\hline \multirow[b]{2}{*}{ Acessos } & \multicolumn{11}{|c|}{ Constituintes do óleo essencial (\%) } & \multirow[b]{2}{*}{$\mathrm{NC}$} \\
\hline & $\mathrm{C} 1$ & $\mathrm{C} 2$ & $\mathrm{C} 3$ & $\mathrm{C} 4$ & $\mathrm{C} 5$ & C6 & $\mathrm{C} 7$ & $\mathrm{C} 8$ & C9 & $\mathrm{C} 10$ & $\mathrm{C} 11$ & \\
\hline AMA & 0,63 & 0,64 & 3,60 & 0,28 & 10,46 & 0,63 & - & 0,70 & 1,44 & 70,77 & 0,48 & 53 \\
\hline API & 1,24 & 0,56 & 0,54 & 3,94 & 76,57 & 0,63 & 0,46 & 0,48 & 1,33 & 0,41 & 0,38 & 67 \\
\hline ARB & 0,14 & - & 2,23 & 0,11 & 30,67 & 0,37 & - & 0,29 & 1,19 & 61,35 & 0,21 & 26 \\
\hline AVB & - & - & 2,09 & - & 37,75 & 0,42 & - & 0,38 & 0,82 & 56,85 & - & 12 \\
\hline AVP & - & 0,14 & 2,21 & 0,15 & 15,63 & 0,37 & - & 0,29 & 0,67 & 76,71 & 0,20 & 24 \\
\hline TR & 13,48 & 18,21 & 24,08 & 24,5 & 28,83 & 26,69 & 28,46 & 28,5 & 30,82 & 34,08 & 39,51 & \\
\hline
\end{tabular}

$\mathrm{C} 1$ = terpinen-4-ol; $\mathrm{C} 2=$ acetato de bornila; $\mathrm{C} 3=(E)$-cariofileno; $\mathrm{C} 4=$ cumarina $\mathrm{I} ; \mathrm{C} 5=$ precoceno $\mathrm{I} ; \mathrm{C} 6=\gamma$-muroleno; $\mathrm{C} 7=\delta$-cadineno; $\mathrm{C} 8$ = $\alpha$-muroleno; $\mathrm{C} 9$ = óxido de cariofileno; $\mathrm{C} 10$ = precoceno II; C11 = 6-hidroximetil-7-metoxi-2,2-dimetil-2H-cromeno; NC = número de compostos; TR = tempo de retenção em min. AMA; API; ARB; AVB e AVP descritos no rodapé da Tabela 1. 


\section{PARTE EXPERIMENTAL}

\section{Obtenção e cultivo dos acessos}

Foram estudados cinco acessos de mentrasto, coletados nos municípios de Mariana-MG (AMA), Piranga-MG (API), Visconde do Rio Branco-MG (ARB) e Viçosa-MG (AVB e AVP). Os acessos do município de Viçosa foram obtidos nas localidades denominadas Bom Sucesso (AVB) e Paraíso (AVP). A identificação específica dos acessos foi feita pelo Dr. J. N. Nakajima (UFU) e as exsicatas encontram-se depositadas no herbário da UFV, sob os números 25.806, 25.807, 25.808, 25.809 e 25.810.

A propagação dos cinco acessos foi realizada por sementes em bandejas de isopor. As mudas foram transplantadas 44 dias após a semeadura. No plantio, realizado em área experimental da UFV, foi adotado o espaçamento de $0,5 \times 0,7$ m e adubação orgânica de três litros de composto por cova.

\section{Delineamento experimental}

O delineamento experimental foi inteiramente casualizado com cinco tratamentos, constituídos pelos cinco acessos de mentrasto. Foram utilizadas três repetições para análise do teor do óleo essencial, sendo cada repetição composta pelo material desidratado.

A coleta de material (parte aérea da planta) para análise do óleo essencial foi realizada aos 84 dias após transplante, na fase de floração. Após a coleta, as plantas foram desidratadas em sala de secagem e mantidas em temperatura ambiente $\left(25-30{ }^{\circ} \mathrm{C}\right)$.

\section{Extração do óleo essencial}

A extração do óleo essencial foi realizada por hidrodestilação no Laboratório de Análise e Síntese de Agroquímicos do Departamento de Química da UFV. Amostras da planta desidratada (em torno de $18 \mathrm{~g}$ ) foram transferidas para um balão, que foi acoplado ao clevenger e este, a um condensador. Após destilação por $2 \mathrm{~h}$ foram recolhidos, aproximadamente, $400 \mathrm{~mL}$ de hidrolato (água + óleo).

O óleo foi extraído da fase aquosa com funil de separação, utilizando o pentano como solvente. Foram realizadas quatro extrações com $40 \mathrm{~mL}$ de pentano. Foi adicionado sulfato de magnésio anidro, em excesso, à fase orgânica, com o objetivo de remover a água presente. A fase orgânica foi filtrada e o pentano removido em evaporador rotativo.

\section{Composição do óleo essencial}

\section{Cromatografia gasosa}

O óleo essencial foi analisado por cromatografia em fase gasosa (CG) em equipamento Shimadzu, modelo $17^{\mathrm{A}}$, equipado com detector de ionização de chama de hidrogênio e coluna capilar SBP - 5 $(30 \mathrm{~cm}$ de comprimento e 0,25 mm de diâmetro interno). O gás de arraste utilizado foi o nitrogênio. A temperatura inicial da coluna foi de $60{ }^{\circ} \mathrm{C}$, sendo programada para ter acréscimos de $3{ }^{\circ} \mathrm{C}$ a cada min, até atingir a temperatura máxima de $240{ }^{\circ} \mathrm{C}$. As temperaturas do injetor e do detector foram fixadas em 220 e $240{ }^{\circ} \mathrm{C}$, respectivamente. Uma quantidade de $10 \mathrm{mg}$ das amostras foram diluídas em $1 \mathrm{~mL}$ de pentano, sendo injetado $1 \mu \mathrm{L}$ da mistura pentano + óleo essencial.

\section{Cromatografia gasosa acoplada ao espectrômetro de massas}

Na identificação dos compostos do óleo essencial, a cromatografia gasosa acoplada ao espectrômetro de massas é um dos métodos mais indicados ${ }^{21,22}$.

A identificação dos compostos do óleo essencial foi realizada por cromatografia gasosa acoplada ao espectrômetro de massas (CG/EM), em equipamento Shimadzu, modelo CG 17A, com detector seletivo de massa, modelo QP 5000 (Shimadzu). A coluna cromatográfica utilizada foi do tipo capilar de sílica fundida com fase estacionária DB 5 , de $30 \mathrm{~mm}$ de comprimento e $0,25 \mathrm{~mm}$ de diâmetro interno, utilizando hélio como gás carreador. As temperaturas foram de $220{ }^{\circ} \mathrm{C}$ no injetor e $300{ }^{\circ} \mathrm{C}$ no detector. A temperatura do forno foi programada de 60 a $240{ }^{\circ} \mathrm{C}$, com acréscimo de $3{ }^{\circ} \mathrm{C}$ a cada min.

A identificação dos compostos foi feita por comparações dos espectros de massas com os espectros existentes no banco de dados do equipamento, com a literatura e pelo índice de Kovat's ${ }^{25,26}$.

\section{Análise estatística}

Os dados foram interpretados por meio de análise de variância e as médias foram comparadas utilizando-se o teste de Tukey a 5\% de probabilidade.

A análise estatística foi feita no programa SAEG (Sistema para Análises Estatísticas e Genéticas).

\section{AGRADECIMENTOS}

Os autores agradecem o apoio do Conselho Nacional de Desenvolvimento Científico e Tecnológico (CNPq), da Fundação Estadual Norte Fluminense (FENORTE) e do Parque de Alta Tecnologia do Norte Fluminense (TECNORTE). Também agradecem ao Prof. A. J. Demuner pela contribuição.

\section{REFERÊNCIAS}

1. Brown Jr., K. S.; Acta Amazônica 1988, 18, 291.

2. Pires, M. J. P.; Gripp, A.; Acta Amazônica 1988, 18, 61.

3. Almeida, V. P.; Figueiredo-Ribeiro, R. C. L.; Revista Brasil. Bot. 1986, 9, 75 .

4. Haslam, E.; Chemistry of vegetable tannins, Academic: London, 1966.

5. Hay, R. K. M.; Svoboda, K. P. Em Volatile oil crops: their biology, biochemistry and production; Hay, R. K. M.; Waterman, P. G., eds.; Longman Group; England, 1993, p. 5.

6. Erickson, R. E.; Lloydia 1976, 39, 8 .

7. Friedrich, H.; Lloydia 1976, 39, 1 .

8. Hefendehl, F. W.; Murray, M. J.; Lloydia 1976, 39, 39.

9. Loayza, I.; Abujder, D.; Aranda, R.; Jakupovic, J.; Collins, G.; Deslauriers, H.; Jean, F.; Phytochemistry 1995, 38, 381.

10. Waterman, P. G.; Em ref. 5, p. 47.

11. Siebertz, R.; Proksch, P.; Witte, L.; Phytochemistry 1990, 29, 2135.

12. Bowers, W. S.; Ohta, T.; Cleere, J. S.; Marsella, P. A.; Science 1976, 193, 542.

13. Abena, A. A.; Ouamba, J. M.; Keita, A.; Phytother. Res. 1996, 10, 164.

14. Magalhães, J. F. G.; Viana, C. F. G.; Aragão Jr., A. G. M.; Morais, V. G.; Ribeiro, R. A.; Vale, M. R.; Phytother. Res. 1997, 11, 183.

15. Ladeira, A. M.; Zaidan, L. B. P.; Figueiredo-Ribeiro, R. C. L.; Hoehnea 1987, 15,53

16. Ming, L. C.; Em Perspectives on new crops and new uses; Janick, J., ed.; ASHS: Alexandria, 1999, p. 469.

17. Marques Neto, J. F.; Costallat, L. T. L.; Fernandes, S. R. M.; Napoli, M. D. M.; Samara, A. M.; Rev. Bras. Reumatol. 1988, 28, 109.

18. Oliveira, F.; Lúcia, M.; Garcia, L. O.; Lecta 1993, 11, 63.

19. Basso, F.; Pisante, M.; Basso, B.; Phytother. Res. 1998, 12, S5131.

20. Sharma, S.; Tyagi, B. R.; Naqvi, A. A.; Thakur, R. S.; J. Essent. Oil Res. 1992, $4,411$.

21. Menut, C.; Lamaty, G.; Zollo, P. H. A.; Kuiate, J. R.; Bessiere, J. M.; Flav. Fragr. J. 1993, 8, 1.

22. Okunade, A. D.; Fitoterapia 2002, 73, 1.

23. Wandji, J.; Bissangou, M. F.; Ouambra, J. M.; Silou, T.; Fitoterapia 1996, $67,427$.

24. Buchanan, B. B.; Gruissem, W.; Jones, R. L; Biochemistry e molecular biology of plants, American Society of Plant Physiologists: Maryland, 2000.

25. Adams, R. P.; Identification of essencial oil components by gas chromatography/mass spectroscopy, Allured Publishing Corporation: Ilinois, 1995.

26. Collins, H. C.; Braga, G. L.; Bonato, P. S.; Introdução a métodos cromatográficos, Unicamp: Campinas, 1997. 